\title{
Pilot Mental Workload with Predictive System Status Information*
}

\author{
Anna C. Trujillo \\ Crew/Vehicle Integration Branch \\ NASA Langley Research Center
}

\begin{abstract}
Research has shown a strong pilot preference for predictive information of aircraft system status in the flight deck. However, the mental workload associated with using this predictive information has not been ascertained. The study described here attempted to measure mental workload. In this simulator experiment, three types of predictive information (none, whether a parameter was changing abnormally, and the time for a parameter to reach an alert range) and four initial times to a parameter alert range $(1$ minute, 5 minutes, 15 minutes, and ETA+45 minutes) were tested to determine their effects on subjects' mental workload. Subjective workload ratings increased with increasing predictive information (whether a parameter was changing abnormally or the time for a parameter to reach an alert range). Subjective situation awareness decreased with more predictive information but it became greater with increasing initial times to a parameter alert range. Also, subjective focus changed depending on the type of predictive information. Lastly, skin temperature fluctuated less as the initial time to a parameter alert range increased.
\end{abstract}

\section{Introduction}

Documented instances exist where some type of early notification to the flight crew of a system parameter deviation could have prevented or lessened the consequences of an aircraft's system failure [1], [2]. This early notification may improve decision making by allowing the flight crew to be more informed, thus decreasing their perceived workload [3]-[5]. Current research has shown the potential benefits that predicting system failures would bring to increasing the safety of flight [6]-[8].

Earlier research has indicated a strong pilot preference for predictive information [6], and other related research has demonstrated that pilots do use the predictive information to affect the alert and its consequences [8]. For instance, with predictive information (in the form of knowing that a parameter was moving abnormally or of knowing the time to an alert [7]), pilots retrieved checklists, descended, diverted, and declared emergencies earlier [8]. These results also indicated that the longer the time pilots had to deal with an emerging problem, the earlier they performed various actions [8].

The possible changes in mental workload a pilot might experience when using predictive information have not yet been fully analyzed. Currently, flight crews have defined procedures that they follow when a parameter reaches an alert range but no such procedures exist regarding a parameter moving towards an alert range. Therefore, it was of interest to determine if predictive information would reduce mental workload by reducing the time stress of handling failures once the parameter reached an alert range or if it would increase mental workload by introducing additional information and tasks. Thus, this paper presents the results from an investigation of the mental workload of pilots exposed to various levels of predictive information.

\subsection{Objectives}

This experiment was conducted to determine the mental workload associated with using predictive information in an operational setting during non-normal system events. Mental workload was defined by and measured by four methods in this experiment: (1) NASA-TLX self-reported perceived workload ratings (workload) [9], (2) self-reported perceived situation awareness (SA) ratings, (3) self-reported perceived focus of attention (focus), and (4) skin temperature (temperature). High mental workload was defined as a high NASA-TLX self-reported perceived workload rating, low self-reported perceived SA ratings (possibly indicative of narrowed focus or concentration on one aspect of a problem), and low skin temperature, which typically connotes higher stress levels [10]. The data collected addressed how three types of predictive information, four initial times to a parameter alert range, and subjects' actions affected mental workload.

* To be published in Fourth Symposium On Human Interaction With Complex Systems, $\mathrm{OH}, \mathrm{March} 22-24,1998$. 


\subsection{Experimental variables}

Of the four experimental variables, two were directly manipulated: the predictive information available and the initial time to a parameter alert range. The predictive information available, a between-subject variable, was one of three types: (1) none (baseline), (2) whether a parameter was increasing or decreasing abnormally (direction), or (3) the time remaining to a parameter alert range (countdown). The second variable was the initial time to a parameter alert range, a within-subject variable, and it had four levels: (1) 1 minute, (2) 5 minutes, (3) 15 minutes, and (4) ETA+45 minutes (Estimated Time to Arrival). Baseline predictive information and ETA+45 minutes initial time to a parameter alert range, which placed the alert beyond the end of the flight, were control conditions. The third experimental variable was the four independent faults each subject encountered, and was partially controlled in that the parameter would degrade in a regulated manner. The last experimental variable, action performed, was calculated based on when subjects performed certain actions, such as diverting, and was used in the data analysis for SA ratings, focus, and temperature.

1.2.1. Predictive information available. In the baseline condition, no predictive information was available. Thus, when a parameter reached an alert range, the subjects saw the typical alert message (e.g., CABIN ALT) with the accompanying aural alert (table 1). In the other two conditions, direction and countdown, a text message on the alerting system screen notified subjects that a parameter was moving towards an alert range once a failure occurred. For the direction condition, subjects were told that a parameter was increasing or decreasing abnormally (table 1). For the countdown condition, subjects were told when a parameter would reach its alert range for the current aircraft state (table 1). The onset of an alert was updated in increments of whole minutes if the time remaining was greater than 1 minute. If the onset of an alert was less than $60 \mathrm{sec}$, the message updated for every 15 -sec change in the onset of an alert.

Table 1 - Examples of Predictive Information

\begin{tabular}{|l|l|l|}
\hline \multicolumn{1}{|c|}{ Condition } & \multicolumn{1}{|c|}{$\begin{array}{c}\text { Predictive } \\
\text { Information }\end{array}$} & $\begin{array}{c}\text { Alert } \\
\text { Information }\end{array}$ \\
\hline \hline Baseline & None & "CABIN ALT" \\
\hline Direction & "CABIN ALT INC" & "CABIN ALT" \\
\hline Countdown & "CABIN ALT 7MIN" & "CABIN ALT" \\
\hline
\end{tabular}

In all cases, the information presented to subjects was always correct and accurate. The predictive information had an alert category of advisory. Furthermore, parameters increased or decreased at a constant rate dependent on the state of the aircraft. Lastly, in the direction and countdown condition, the related standard alert information message replaced the predictive information message when the parameter reached an alert range.

1.2.2. Initial time to a parameter alert range. Each subject saw four initial times to a parameter alert range (the time interval to an alert once a failure began): (1) 1 minute, (2) 5 minutes, (3) 15 minutes, and (4) ETA+45 minutes. The configuration of the aircraft affected the onset of an alert; for example, throttling back the engine during the scenario in which the EGT (Exhaust Gas Temperature) increased would delay the onset of an alert.

1.2.3. Faults. All of the data runs, or scenarios, and the training runs included a fault in which a parameter would eventually reach an alert range if the subject took no action. The faults were (1) cabin altitude increase, (2) forward cargo overheat, (3) EGT increase, and (4) oil quantity decrease. The training run was an avionics overheat. All failures were designed to behave as realistically as possible [11]-[14] and are described below.

For the scenario with the cabin altitude increase, the cabin altitude increased to the airplane altitude. The outflow valve, if checked, was in the fully closed condition once the failure started. Although the increase could not be controlled through the environmental system, the cabin altitude warning would not be reached if the subject descended below 10,000 ft mean sea level and if he had at least $4 \frac{1}{2}$ minutes until the alert range was to be reached - the time needed to descend from the initial altitude of $37,000 \mathrm{ft}$ to $10,000 \mathrm{ft}$.

In another data run, the forward cargo hold, initially set-up for carrying animals, had a temperature increase until it reached the fire warning limit. If the subject changed to the cargo mode, the temperature increase would slow due to the lower temperature setting. Also, if he discharged the forward cargo fire bottle before the alert range was reached, the forward cargo temperature would never reach the alert range. If the subject discharged the forward cargo fire bottle after the fire warning, as the forward cargo fire checklist instructs him to do, the temperature would drop below the alert range.

During the scenario with the EGT increase, the EGT rose steadily and if it reached the alert range, the subject would have to follow the engine failure/shutdown procedure. The increase could be slowed if the subject throttled back the engine with the increasing EGT or stopped if the affected engine was shut down. If the subject restarted the engine, the EGT would again increase until it reached the alert range. 
Finally, one scenario resulted in an oil quantity decrease due to loss of oil. The oil pressure triggered the alert once it reached an alert range. The only way to decrease the rate of oil loss was to shut down the affected engine.

For the avionics overheat training run, changing the avionics mode to override from its initial position of normal would decrease the rate of temperature increase. Furthermore, by disconnecting bus 3, the temperature would stay below the warning limit. Thus, the load on bus 3 was the primary cause of the overheat.

1.2.4. Actions performed. For data analysis, each data run for each subject was parsed into no more than four distinct segments. Subjects were not penalized in the data analysis if they did not perform a particular segment. "Notice problem" was from the beginning of the scenario to when the subject first noticed a problem. Retrieving the appropriate checklist, which all failures had, or trying to affect the time to an alert signaled problem detection. If the subject first brought up a checklist, then "next action" was defined from noticing the problem to the subject's affecting the onset of an alert. The subsequent action, if done, was "to divert" and was defined from checklist retrieval or alteration of the onset of an alert to diversion time. The last segment, "after divert," was from the last action the subject did.

The specific actions considered as trying to affect the onset of an alert depended on the scenario. These actions were checking the outflow valve position for the cabin altitude increase failure, changing the forward cargo compartment mode from animal to cargo or discharging a forward cargo compartment fire bottle for the forward cargo fire scenario, and throttling back or shutting down the affected engine for the EGT increase and oil quantity decrease scenarios.

Since the flight was under ETOPS (Extended Twinengine OPerationS) rules, subjects had to divert for the oil quantity decrease and EGT increase failures when they shut down an engine. The ETOPS rules do not specify a diversion is necessary with cabin pressure loss, but for fuel efficiency reasons and passenger comfort, the logical choice would be to divert. If a subject had a forward cargo fire warning, he would be required to divert under ETOPS rules. If a subject discharged the fire bottle before the fire warning, thus preventing the temperature from increasing into the alert range, he did not technically have to divert but prudence recommended diverting anyhow because of the strong possibility of fire.

\section{Experiment design}

\subsection{Subjects}

Twelve glass-cockpit airline pilots familiar with ETOPS rules participated as subjects. Seven were currently first officers, the remaining five were captains. The average age was 48 years old and the average commercial airline flight experience was 16 years.

\subsection{Test design}

This experiment was run in the Advanced Civil Transport Simulator at the NASA Langley Research Center. This simulator had flight performance characteristics similar to a Boeing 757. The flight deck resembled a Boeing 747-400 or MD-11. The subject acted as captain, pilot-not-flying. A confederate first officer (F/O) was pilot-flying and he was well versed in the operation of the simulator. A confederate air traffic controller (ATC) and company dispatch operator provided the necessary coordination with the ground.

The flight was from Dulles airport to Charles de Gaulle airport with a 60-minute ETOPS rule; i.e., the plane was never more than 60 minutes from an alternate airfield. The 60-minute rule was used in order to have several PETs (Point of Equal Time); i.e., the point where the plane was 60 minutes from any suitable alternate airport. The scenarios were set up such that each segment of flight started before a PET; thus, this experiment only included the cruise phase of flight. If the configuration of the aircraft did not change during the fault, the affected parameter would reach an alert range a few minutes before the aircraft intersected the PET except in the ETA+45 minute condition.

Any materials and information the subject needed were provided to him. Plotting charts, landing plates, a dispatch weather briefing, and a flight plan were available in paper form. Checklists were electronic and mimicked the Boeing model of the quick reference handbook [15]. Voice communication was used for ATC and dispatch. Both ATC and dispatch were able to supply current weather information at any of the diversion airfields. Basically, the weather at all diversion airfields was acceptable for landing-drizzle with a ceiling around $1,000 \mathrm{ft}$ and visibility approximately $1 \frac{1 / 2}{2}$ miles with winds at no more than 10 knots. ATC also reasonably expedited (within $15 \mathrm{sec}$ ) any requests subjects had regarding course changes. The confederate $\mathrm{F} / \mathrm{O}$ was able to answer 
operational questions from the subject; i.e., he supplied all the operational information normally found in the aircraft manual. Lastly, subjects made any passenger announcements or held conferences with the head flight attendant, or purser, to the experimenter sitting in the back of the simulator.

As mentioned earlier, the faults, the initial times to a parameter alert range, and actions performed were withinsubject variables while the predictive information was a between-subject variable. Since subjects could only see each failure once, each subject had four data runs in addition to a training run. Thus, the overall result was that all subjects saw each of the four faults once and each of the four initial times to an alert once with only one of the three types of predictive information.

\subsection{Dependent measures}

The dependent measures consisted of workload ratings after each data run, and SA ratings, focus and skin temperature measurements during each scenario. The workload ratings consisted of a subject's rating of his perceived mental, physical, and temporal demand; performance; effort; and frustration with workload increasing as the rating increased [9]. SA was broken down into spatial, mission, and system awareness. Subjects rated their perceived three SAs on a rating scale of 1 to 5, with 1 as low awareness and 5 as high awareness. Spatial awareness was defined as knowing the altitude and aircraft position (lateral, vertical, and time axis) relative to desired flight path and relative to other aircraft, terrain, weather, suitable alternates, routes, etc. Mission awareness encompassed flight planning (fuel, weight, alternates, winds, efficiency items), external factors (ATC procedures, delays, traffic, weather), and contingency plans to solve operational and safety problems. Lastly, systems awareness was defined as knowledge about the basic operation of systems, faults and failures, non-normal procedures, and fault consequences. Focus was defined as the SA category (spatial, mission, system) the subject felt he was currently concentrating on.

Peripheral skin temperature was sensed with a 1-sec time constant thermistor attached to the dorsal surface of the index finger of the left hand and held in place by medical adhesive tape. The temperature was conditioned by the corresponding $\mathrm{J} \& \mathrm{~J}$ Engineering module and acquired using the J\&J I-330 interface with a Compu-Add 386 computer operating under the J\&J "USE" software system. The application was programmed so that each stored data point was an average of $5 \mathrm{sec}$ of activity.

\subsection{Procedure}

When a subject first arrived, he received an overview of this experiment including instructions about the workload questionnaire and SA definitions. After this introduction, the confederate $\mathrm{F} / \mathrm{O}$ gave a detailed description of the simulator and its operation, and the flight plan to the subject before the training run started. The training run included the avionics overheat fault. For the training run, the initial time to a parameter alert range was 5 minutes given the initial aircraft configuration. No data were recorded during training.

A short break was taken after the training run and before data run 1. An hour lunch break followed the first data run. After lunch, the subject completed data runs 2 through 4. Each data run took approximately 30 minutes. During each data run, awareness ratings and focus were requested every 5 minutes and peripheral skin temperature was an average temperature over $5 \mathrm{sec}$ increments.

At the end of each data run, the subject was asked about the failure, his actions, and his workload. The presentation order of predictive information and initial time to a parameter alert range were counterbalanced while scenario order was only partially balanced due to the number of subjects.

\subsection{Data analysis}

The six individual workload ratings were normalized on a scale from 0 to 100 with 0 as no workload and 100 as maximum workload. They were combined into an average workload rating for each subject by data run. These average normalized workload ratings were then analyzed using the analysis of variance procedure in SPSS $®$ [16].

The SA ratings and temperature data were analyzed using the "General Linear Model" with an F-test in SPSS ${ }^{\circledR}$ [16]. Focus was analyzed using the "KruskalWallis H" model with a Chi-square test $\left(\chi^{2}\right)$ in SPSS ${ }^{\circ}$ [16] since the data were not continuous and could not be averaged without loss of meaning. Temperature was normalized for each subject by data run by subtracting from each temperature measurement the average temperature before the failure was noticed. This was done in order to analyze all data on the same scale. Also, due to temperature data collection problems, temperature data only existed for 3 subjects in the baseline condition, 4 subjects in the direction condition, and 2 subjects in the time condition.

In the analysis of the data, significance (for both twotailed tests, $\mathrm{F}$ and $\chi^{2}$ ) was defined as a p-value less than 
0.05. Also, for main-order effects, a Tukey Honestly Significant Difference (HSD) post hoc test was done [17].

\section{Results}

\subsection{Predictive information}

As was also reported in [8], predictive information was significant for workload $(\mathrm{F}(2,24)=3.72, \mathrm{p}<0.04)$. Workload was rated significantly lower for the familiar baseline condition than the direction condition while the countdown condition was not statistically different from either one (table 2).

Table 2 - Workload Ratings

\begin{tabular}{|l|cc|}
\hline \multicolumn{1}{|c|}{ Factor } & Mean & Std Dev \\
\hline \hline Predictive Information & & \\
Baseline $^{*}$ & 28 & 15 \\
Direction* $^{*}$ & 43 & 18 \\
Countdown & 40 & 14 \\
Initial Time to a Parameter & & \\
Alert Range & & \\
1 min & 41 & 19 \\
5 min & & 19 \\
15 min & 41 & 9 \\
ETA+45 min $^{*}$ & 41 & 13 \\
\hline
\end{tabular}

Note: $0=$ low workload, $100=$ high workload *=significantly different from one another

Analysis of the SA measures indicated an interaction between predictive information and type of SA measure (spatial, mission, and system) $(\mathrm{F}(4,529)=3.22, \mathrm{p}<0.02)$. Spatial awareness had approximately the same relatively high rating for all three types of predictive information (fig. 1). Mission awareness was slightly less for the direction predictive information while system awareness was less for both the direction and countdown predictive information compared to the baseline condition (fig. 1).

$\chi^{2}$ tests revealed that predictive information $\left(\chi^{2}(2)=14.85, p<0.01\right)$ was significant for focus (table 3 ). In order to easily compare the number of times subjects focused on the three SA categories, the ratio of area of focus across predictive information was graphed (fig. 2). As can be seen in fig. 2, focus changed from spatial in the baseline condition to mission with the direction and countdown predictive information while system awareness stayed approximately even for all three types of predictive information.

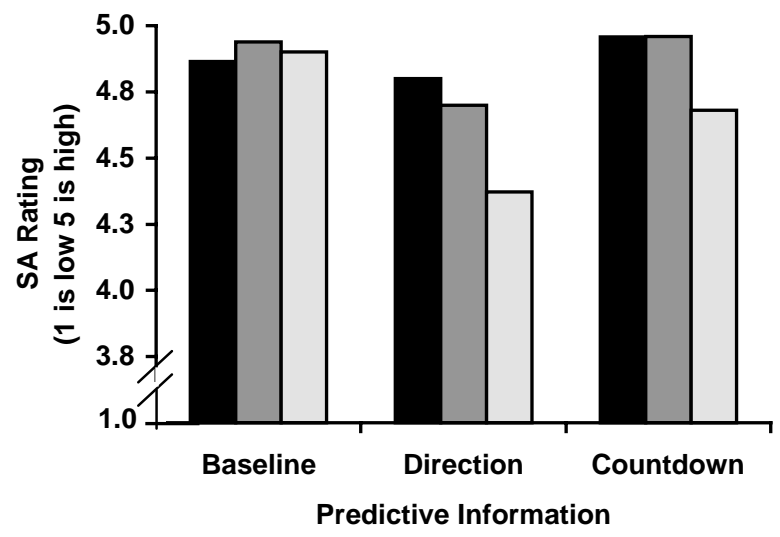

Figure 1 - Interaction between Predictive Information and SA Type

SA Type: $\square$ Spatial $\square$ Mission $\square$ System

Table 3 - Focus

\begin{tabular}{|c|c|c|c|c|}
\hline Factor & \multicolumn{3}{|c|}{$\%$ of Times Focus Was } & $\begin{array}{c}\text { Total } \\
\text { Number }\end{array}$ \\
\hline $\begin{array}{l}\text { Predictive } \\
\text { Information }\end{array}$ & & & & \\
\hline Baseline & 49 & 22 & 28 & 81 \\
\hline Direction & 11 & 54 & 34 & 70 \\
\hline $\begin{array}{l}\text { Countdown } \\
\text { Action }\end{array}$ & 17 & 49 & 34 & 71 \\
\hline Notice Problem & 40 & 29 & 31 & 83 \\
\hline Next Action & 23 & 38 & 38 & 26 \\
\hline To Divert & 10 & 33 & 57 & 30 \\
\hline After Divert & 22 & 57 & 22 & 83 \\
\hline
\end{tabular}

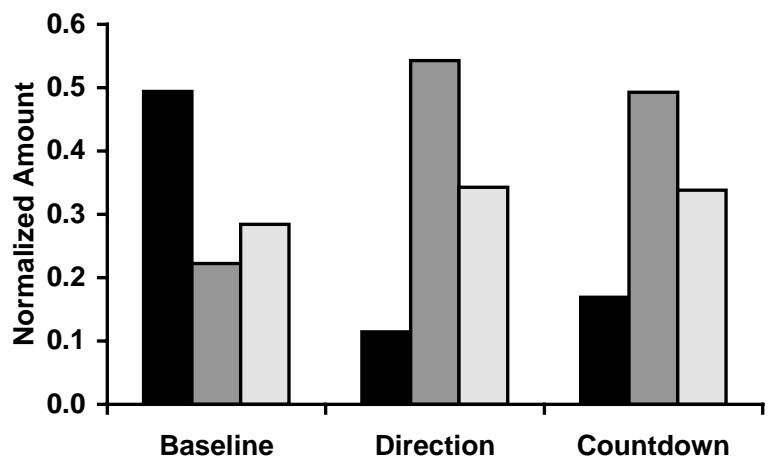

Figure 2 - Ratio of Focus by Predictive Information

SA Type: $\square$ Spatial $\square$ Mission $\square$ System


Figure 3 shows normalized temperature as it varies with action taken and predictive information. Temperature first rose and then decreased for the baseline and countdown conditions. The countdown predictive information had the greatest temperature drop (indicating higher stress), which corresponds to its lower SA and high workload ratings.

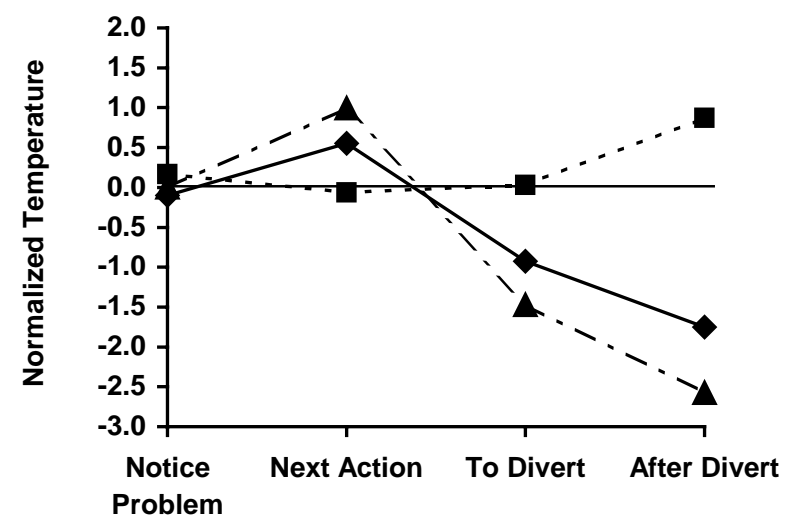

Figure 3 - Normalized Temperature for Action by Predictive Information

Predictive Information: — Baseline

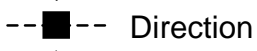

$-\Delta-$ - Countdown

\subsection{Initial time to a parameter alert range}

Also reported in [8], the initial time to a parameter alert range was significant for workload $(\mathrm{F}(3,24)=3.38$, $\mathrm{p}<0.04)$. As expected, the ETA+45 minute initial time to a parameter alert range had the lowest workload rating (table 2). This was because subjects did not have to confront the failure, if they even noticed the problem, since the alert was going to occur after landing. No difference in workload ratings among the other three initial times to a parameter alert range was present and were all higher than the ETA+45 minute condition.

SA measures were also significant $(F(3,529)=3.35$, $\mathrm{p}<0.02)$. The 5-minute initial time to a parameter alert range had a slightly lower SA rating than the 1-minute initial time to a parameter alert range (table 4). The 15and ETA+45 minute initial times to a parameter alert range were not significantly different from either the 1- or 5-minute initial time to a parameter alert range (table 4).
Table 4 - SA for Initial Time to an Alert

\begin{tabular}{|c|ccc|}
\hline $\begin{array}{c}\text { Initial Time to a } \\
\text { Parameter Alert Range }\end{array}$ & $\mathbf{N}$ & Average & Std Dev \\
\hline \hline 1 minute $^{*}$ & 156 & 4.88 & 0.41 \\
5 minutes $^{*}$ & 156 & 4.73 & 0.61 \\
15 minutes & 195 & 4.77 & 0.54 \\
ETA+45 minutes & 161 & 4.84 & 0.42 \\
\hline
\end{tabular}

Note: $1=$ low SA, 5=high SA

*=significantly different from one another

Figure 4 shows normalized temperature as it varies with action taken and initial time to a parameter alert range. The 5-minute initial time to a parameter alert range had the largest temperature decrease. Basically, the temperature fluctuations became less as the initial time to a parameter alert range increased.

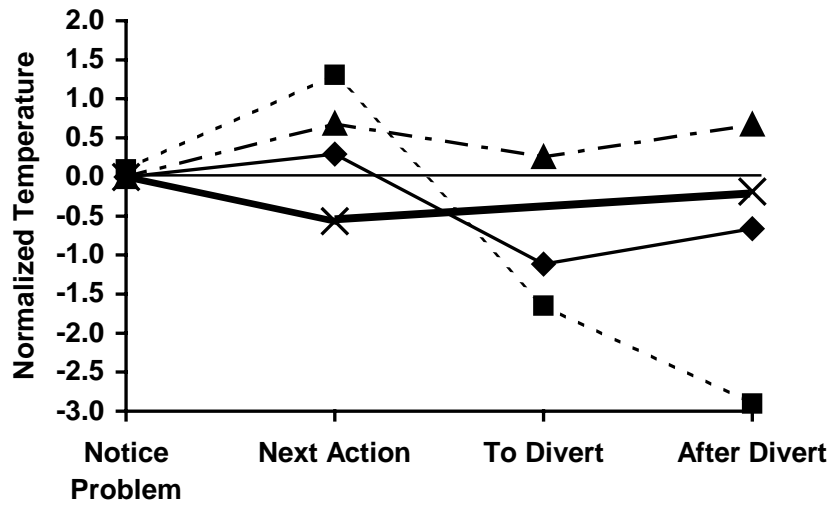

Figure 4 - Normalized Temperature for Action by Initial Time to Parameter Alert Range

Initial Time to

Parameter Alert Range:

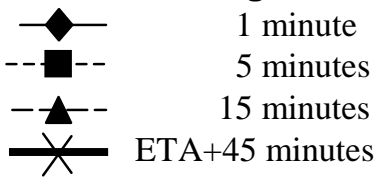

\subsection{Actions}

$\chi^{2}$ tests indicated that actions performed $\left(\chi^{2}(3)=10.36, p<0.02\right)$ was significant for focus (table 3$)$. As with predictive information, in order to easily compare the number of times subjects focused on the three SA categories, the ratio of area of focus across action was graphed (fig. 5). Subjects focused more on the system before ("next action") and during diverting ("to divert") and then they focused on the mission after diverting. 


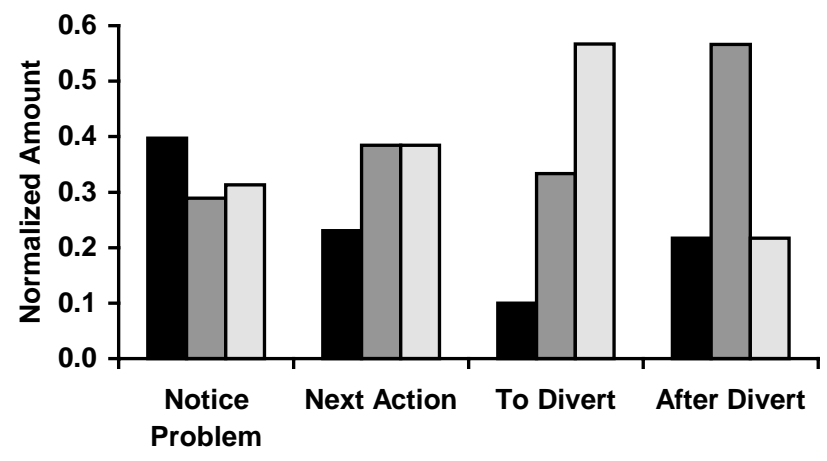

Figure 5 - Ratio of Focus by Action SA Type: $\square$ Spatial $\square$ Mission $\square$ System

\section{Discussion}

To identify the mental workload associated with using predictive information, a simulator experiment testing three types of predictive information, four initial times to a parameter alert range, and subjects' actions was conducted. The three types of predictive information were: (1) baseline, (2) direction, and (3) countdown, and the four initial times to a parameter alert range were: (1) 1 minute, (2) 5 minutes, (3) 15 minutes, and (4) ETA+45 minutes. Both of these variables were found to affect subjects' mental workload.

The direction and countdown predictive information had higher workload ratings associated with them than the baseline information. The SA data indicated that direction predictive information had the greatest decrease in SA, especially system SA, which is, not surprisingly, where the system failures had the most effect initially. Thus, predictive information, because it was unfamiliar information, increased workload and lowered SA. The increase in workload and decrease in system SA scores for direction predictive information may have been partially due to subjects having to estimate how much time they had before an alert range would be reached. Unlike the countdown condition, the only way to do this was to approximate the parameter's rate of change.

However, the greatest contributor to workload and the difference in system SA measures appears to be choosing which actions to carry out. Subjects knew there was a problem because of the predictive information but they had to figure out what was causing it in order to plan the best course of action. Furthermore, no procedures were given regarding the use of direction and countdown predictive information and this, most likely, accounted for subjects rating workload for countdown predictive information closer to direction predictive information than to the baseline condition. Apparently, deciding on the proper course of action for the direction and countdown conditions increased workload more than estimating the onset of an alert for the direction condition.

Even though predictive information lowered SA, this was not necessarily a negative result. The lower SA could indicate that the subject recognized that he had a problem and needed to spend more resources on it. This was evident in the shift of focus from spatial and system with no predictive information to mission and system with predictive information. Considering subjects were pilots-not-flying, they should have been more concerned about the overall mission and this shift in focus is appropriate. Also, this change in focus from spatial to mission may have also contributed to increasing mental workload for predictive information. Furthermore, as pilots-not-flying, the subjects' primary duties included watching the systems, which is where the failures occurred, and diagnosing the failure. The lower system SA measures reflect these primary duties. Also, subjects focused more on the system before and during diverting and on the mission after diverting. This is not surprising in that subjects first had to figure out what the problem was, the seriousness of it, and then how it was going to affect the mission.

More of a temperature fluctuation existed for the countdown condition and it does closely follow the baseline condition suggesting that subjects could feel as comfortable with countdown predictive information as they do now with no predictive information once they have had some experience using it. The temperature increase associated with the direction predictive information may be a function of stress levels being reduced by the presence of the familiar, and assumed useful, information; whereas the baseline condition was familiar but not as useful as the direction and countdown predictive information.

Except for the 1-minute initial time to a parameter alert range, as the initial times to a parameter alert range increased, SA increased most likely due to having enough time to figure out the proper course of action and the cause of the slow degradation. The 1-minute initial time to a parameter alert range may not have given subjects enough time to diagnose the problem; they may have treated it just like the way they handle alerts currentlyfollow checklist procedures. When subjects had enough time to troubleshoot, i.e., for the 5-, 15-, and ETA+45 minute initial times to a parameter alert range, the SA rating increased as the initial time to an alert increased.

Skin temperature fluctuated less as the longer initial times to a parameter alert range increased suggesting less stress was present since there was time to handle the problem until an alert sounded. The 1- and 5-minute initial times to a parameter alert range show greater temperature fluctuations probably because there is more 
time pressure at later events. The 5-minute initial time to a parameter alert range may have given the subjects a false sense of security while the 1-minute initial time to a parameter alert range may not have given subjects enough time to determine their course of action before the alert was reached resulting in subjects treating the 1-minute condition the same way they treat alerts currently, by reacting to it. Thus, the lower temperature fluctuations for the 15- and ETA+45 minute initial times to a parameter alert range may represent a decrease in stress from current levels, which is also reflected in the increasing SA ratings. Furthermore, previous analysis had suggested that subjects were primed for the failures and made predictions before the alert occurred [8]. Thus, the longer initial times to a parameter alert range can be viewed as providing foreknowledge of a failure to the flight crew.

As for workload ratings, the initial time to a parameter alert range equally affected workload for all the conditions except where the alert occurred after landing. In this case, no actions had to be taken even if the problem was noticed. The workload ratings for the other initial times to a parameter alert range (1, 5, and 15 minutes) were all high but not significantly different from one another most likely because subjects still had to handle the failure and had to decide how to manage the aircraft since no procedures existed for alerts that had yet to occur.

\section{Conclusions}

Based on the above results and discussion, foreknowledge of a parameter reaching an alert range is beneficial. The negative aspects of the information can be overcome by increasing the familiarity of this type of information and providing flight crews with procedures.

Other aspects, though, must be investigated before the full usefulness of predictive information can be understood. Further research into the optimal prediction time, acceptable false alarm rate, and accuracy of the predictive information must be conducted. Also, it would be of benefit to ascertain how useful the information would be when pilots are not primed for a failure. On the more operational side, the ability to reliably estimate rates of change and times to an alert with the false alarm rate and accuracy required by the pilots needs to be investigated before procedures are developed using the time to an alert predictive information.

\section{References}

1. Aircraft Accident Report. Eastern Airlines, Inc., Lockheed L-1011, N334EA, Miami International Airport, Miami, Florida, May 5, 1983. NTSB-AAR-84-04, National Transportation Safety Board, Mar. 1984. (Available from NTIS as PB84 910 404.)

2. Batelle. Aviation Safety Reporting System. Search Request Number 1929, Aircraft Subsystems Failures, Mountain View, California, 1990.

3. McCormick, Ernest James; and Sanders, Mark S.: Human Factors in Engineering and Design. Fifth ed., McGrawHill, 1982.

4. Wickens, Christopher D.: Engineering Psychology and Human Performance. Scott, Foresman \& Co., 1984.

5. Weiner, Earl L.; Kanki, Barbara G.; and Helmreich Robert L., eds.: Cockpit Resource Management. Academic Press, 1993.

6. Trujillo, Anna C.: Effects of Historical and Predictive Information on Ability of Transport Pilot to Predict an Alert. NASA TM-4547, 1994.

7. Trujillo, Anna C.: Airline Transport Pilot Preferences for Predictive Information. NASA TM-4702, 1996.

8. Trujillo, Anna C.: "Pilot Performance With Predictive System Status Information" in 1997 IEEE International Conference on Systems, Man, and Cybernetics: Computational Cybernetics and Simulation. Oct. 1997.

9. Hart, S.G. and Staveland, L.E.: "Development of NASATLX (Task Load Index): Results of Empirical and Theoretical Research," in P.A. Hancock \& N. Meshkati (eds.), Human Mental Workload. Elsevier, 1988.

10. Green, J. and Shellenberger, R.: The Dynamics of Health and Wellness: A Biopsychosocial Approach. Holt, Rinehart and Winston, Inc., 1991.

11. 767 Flight Manual - Handbook, Revision \#72. United Airlines SFOEG - New Aircraft and Operational Engineering. Oct. 1986.

12. Correspondence with Richard Gifford, UAL Pilot, Ret. 1996.

13. Correspondence with Dave Simmon, UAL Pilot, Ret. 1996.

14. Wild, Thomas W.: Transport Category Aircraft Systems. IAP, Inc., 1990.

15. 757 Quick Reference Handbook. The Boeing Company. Feb. 1992

16. SPSS: SPSS ${ }^{\circledR} 7.5$ for Windows ${ }^{\circledR}$. SPSS Inc., 1997.

17. Howell, D.C.: Statistical Methods for Psychology, $2^{\text {nd }}$ ed. Duxbury Press, 1987. 